\title{
Monte Carlo Analysis of the Dynamic Behavior of InAlAs/InGaAs Velocity Modulation Transistors: A Geometrical Optimization
}

\author{
B.G. Vasallo*, T. González, D. Pardo and J. Mateos \\ Universidad de Salamanca, Plaza de la Merced, s/n, 37008, Salamanca, Spain
}

\begin{abstract}
The influence of the geometry on the dynamic behavior of InAlAs/InGaAs velocity modulation transistors is analyzed by means of a Monte Carlo simulator in order to optimize the performance of this new type of transistor. In velocity modulation transistors, based on the topology of a double-gate high electron mobility transistor, the source and drain electrodes are connected by two channels with different mobilities, and electrons are transferred between both of them by changing the gate voltages in differential mode. Consequently, the drain current is modulated while keeping the total carrier density constant, thus in principle avoiding capacitance charging/ discharging delays. However, the low values taken by the transconductance, as well as the high capacitance between the two gates in differential-mode operation, lead to a deficient dynamic performance. This behavior can be geometrically optimized by increasing the mobility difference between the two channels, by increasing the channel width and, mainly, by reducing the gate length, with a higher immunity to short channel effects than the traditional architectures.
\end{abstract}

PACS: 85.30.De, 85.30.Tv

\section{Introduction}

III-V high electron mobility transistors (HEMTs) have shown an excellent performance for high-frequency operation [1]. To further improve their behavior, alternative solutions based on an evolution of the standard HEMT design have been proposed, as the double-gate (DG) HEMT, a HEMT with two gates placed on each side of the conducting InGaAs channel [2-6]. The progress of the DG-HEMT technology has allowed the design and fabrication of III-V velocity modulation transistors (VMTs) $[7,8]$. In VMTs [7-15], the conducting channel is divided into two regions, a high-mobility (high- $\mu$ ) undoped channel and a low- $\mu$ channel obtained by compensated doping $\left(N_{\mathrm{A}}=N_{\mathrm{D}}\right)$, with two (top and bottom) gates controlling the electron density. Carriers are transferred between the two channels by changing the gate voltages $\left(V_{\mathrm{G} 1}\right.$ and $\left.V_{\mathrm{G} 2}\right)$ in differential mode $(\mathrm{DM})$, in which a potential $\pm V_{\mathrm{GDIFF}} / 2$ is added to a bias voltage $V_{\mathrm{GOFF}}$ which adjusts the total channel electron concentration $\left(V_{\mathrm{GDIFF}}=V_{\mathrm{G} 1}-V_{\mathrm{G} 2}\right)$. Thus, the drain current $I_{\mathrm{D}}$ is modulated while keeping constant the total carrier density, and it is in principle possible to overcome the transit-time limit for high-frequency applications. However, the dynamic behavior of the VMT is not as exceptional as expected [9] due to the low values of the transconductance $g_{\mathrm{m}}$, and mainly due to the high capacitance be-

\footnotetext{
* corresponding author; e-mail: bgvasallo@usal.es
}

tween both gates $C_{\mathrm{g} 1 \mathrm{~g} 2}$ [8]. In contrast, this device does not follow the traditional scaling rules for standard field effect transistors (FETs) and provides a high immunity to short-channel effects [8].

In this work, an optimization of the geometry of recessed short-channel InAlAs/InGaAs VMTs is performed by means of an ensemble 2D Monte Carlo (MC) simulator self-consistently coupled with a 2D Poisson solver [16]. This model, which has provided a full microscopic interpretation of its dynamic performance [8], allows a geometrical optimization of VMTs in order to achieve optimal operation frequencies.

\section{Physical model}

The validity of the semiclassical MC model used for the simulation of VMTs has been proved for standard [16] and DG $[5,6]$ HEMTs by reproducing their experimental static and dynamic behavior. This technique has been also successfully applied to the study of the static and dynamic behavior of a fabricated VMT [7, 8].

The topology of the simulated VMT, similar to that of the fabricated transistors, is sketched in Fig. 1. The active layer structure and the technological process for the fabrication were detailed in Ref. [7]. Two opposite gate electrodes control the total electron density in the channels as well as the carrier shift between them in DM operation. The only difference with respect to a DG-HEMT [2-6] is that the channel is divided into two regions: a high- $\mu$ undoped channel and a low- $\mu$ channel 
with compensated doping. The compensated-doping allows increasing the ionized impurity scattering and thus decreasing the electron mobility. Initially, we analyze a VMT in which the gate length is $L_{\mathrm{g}}=100 \mathrm{~nm}$, the channel width $d_{\mathrm{C}}=40 \mathrm{~nm}$, and $N_{\mathrm{A}}+N_{\mathrm{D}}=10^{19} \mathrm{~cm}^{-3}$. Then, similar structures with different geometrical parameters are studied.

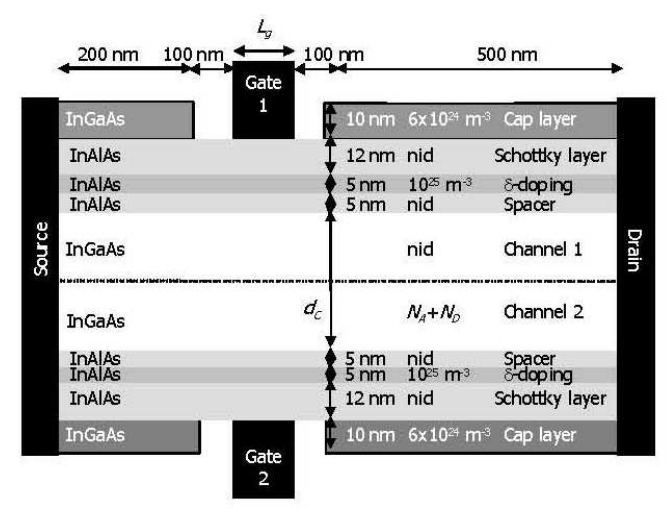

Fig. 1. Schematic topology of the VMT under analysis.

Since the small signal equivalent circuit of VMTs in DM operation is not well established, in order to carry out the analysis of the dynamic behavior of VMTs an input capacitance $C_{\mathrm{IN}}$ is defined as $\operatorname{Im}\left[Y_{11}\right] / 2 \pi f$, being $Y_{11}=\Delta I_{\mathrm{IN}} / \Delta V_{\mathrm{IN}}$ when $V_{\mathrm{DS}}$ remains constant, and $f$ the operation frequency. $C_{\mathrm{IN}}$ can be generally considered as the addition of the gate-to-source $C_{\mathrm{gs}}$, gate-to-drain $C_{\mathrm{gd}}$ and gate1-to-gate $2 C_{\mathrm{g} 1 \mathrm{~g} 2}$ capacitances. When working in $\mathrm{DM}, C_{\mathrm{gs}}$ and $C_{\mathrm{gd}}$ are practically zero, since the amount of electrons under the gate remains practically constant and there is no need for channel charging/discharging, so that $C_{\mathrm{IN}}$ is nearly $C_{\mathrm{g} 1 \mathrm{~g} 2}$. The cut-off frequency $f_{\mathrm{C}}$ can therefore be calculated as $g_{\mathrm{m}} / 2 \pi C_{\mathrm{IN}}$.

\section{Results}

Figure 2 presents the intrinsic MC output characteristics of a VMT with $L_{\mathrm{g}}=100 \mathrm{~nm}, N_{\mathrm{A}}+N_{\mathrm{D}}=10^{19} \mathrm{~cm}^{-3}$, and $L_{\mathrm{C}}=40 \mathrm{~nm}$ : (a) $I_{\mathrm{D}}-V_{\mathrm{DS}}$ for $V_{\mathrm{GDIFF}}=0 \mathrm{~V}$ (common-mode $\mathrm{CM}$ operation), and (b) $I_{\mathrm{D}}-V_{\mathrm{GDIFF}}$ for $V_{\mathrm{DS}}=0.5 \mathrm{~V}(\mathrm{DM})$, for different values of $V_{\mathrm{GOFF}}$. While in $\mathrm{CM}$ (Fig. 2a) the VMT works as a classic FET device (specifically as a DG-HEMT [2-6]), in differential-mode operation the values taken by $I_{\mathrm{D}}$ depend on $V_{\mathrm{GDIFF}}$ due to the velocity-modulation effect: when increasing $V_{\mathrm{GDIFF}}$ the electron density is transferred from the low- $\mu$ to the high- $\mu$ channel, thus increasing the drain current. MC profiles of electron concentration and mean velocity in both high- $\mu$ and low- $\mu$ channels for different biasings demonstrate the velocity-modulation operation of the proposed transistor $[7,8]$. Concerning the dynamic performance of the $100 \mathrm{~nm}$ gate device, MC results show that the cut-off frequencies take values much lower than predicted [9] (the maximum value of $f_{\mathrm{C}}$ estimated with the MC model is of the order of $200 \mathrm{GHz}$ for a geometry similar to that of the experimental device [8]) because of the low $g_{\mathrm{m}}$ associated to the VM behavior and, remarkably, to the high geometrical capacitance existing between the two gate electrodes when operating in DM, which is the main contribution to $C_{\mathrm{IN}}[7,8]$. To improve the frequency operation, the VMT geometry must be optimized for an enhanced $g_{\mathrm{m}}$ and reduced $C_{\mathrm{IN}}$.

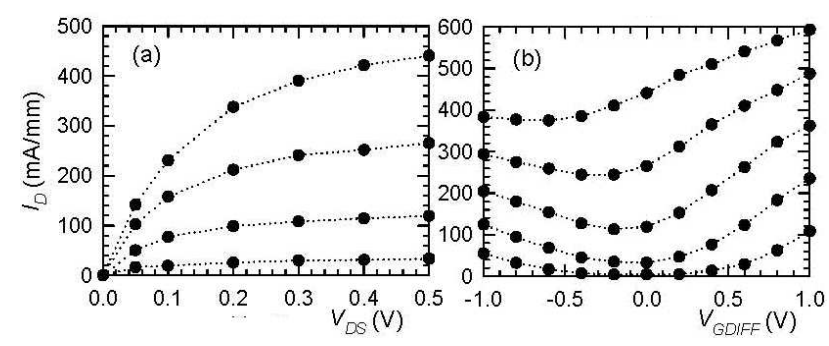

Fig. 2. $\quad \mathrm{MC}(\mathrm{a}) I_{\mathrm{D}}-V_{\mathrm{DS}}$ for $V_{\mathrm{GDIFF}}=0 \mathrm{~V}$ and (b) $I_{\mathrm{D}^{-}}$ $V_{\mathrm{GDIFF}}$ for $V_{\mathrm{DS}}=0.5 \mathrm{~V}$. $V_{\mathrm{GOFF}}$ is $-0.1 \mathrm{~V}$ for the top curves, and the potential step is $\Delta V_{\mathrm{GOFF}}=0.1 \mathrm{~V} . L_{\mathrm{g}}=$ $100 \mathrm{~nm}, d_{\mathrm{C}}=40 \mathrm{~nm}$ and $N_{\mathrm{A}}+N_{\mathrm{D}}=10^{19} \mathrm{~cm}^{-3}$.

The value $g_{\mathrm{m}}$ can be improved by increasing the difference in electron velocity between both channels, which can be achieved by rising the compensated doping $N_{\mathrm{A}}+N_{\mathrm{D}}$. Figure 3 presents the MC values of (a) $g_{\mathrm{m}}, C_{\mathrm{IN}}$, and (b) $f_{\mathrm{C}}$, as a function of $N_{\mathrm{A}}+N_{\mathrm{D}}$ for $V_{\mathrm{GDIFF}}=0 \mathrm{~V}$, $V_{\mathrm{DS}}=0.5 \mathrm{~V}$ and $V_{\mathrm{GOFF}}$ providing the maximum value of $f_{\mathrm{C}}$. As expected, $g_{\mathrm{m}}$ is improved when increasing $N_{\mathrm{A}}+N_{\mathrm{D}}$, while $C_{\mathrm{IN}}$ remains almost constant. However, for a value of $N_{\mathrm{A}}+N_{\mathrm{D}}=5 \times 10^{19} \mathrm{~cm}^{-3}, \mu$ in the low- $\mu$ channel is so small $\left(\approx 840 \mathrm{~cm}^{2} /(\mathrm{V} \mathrm{s})\right)$ that a further rise in the value of the compensated doping does not lead to an improvement in $f_{\mathrm{C}}$. On the contrary, it degrades the charge transfer between channels (lower value in $g_{\mathrm{m}}$ ).

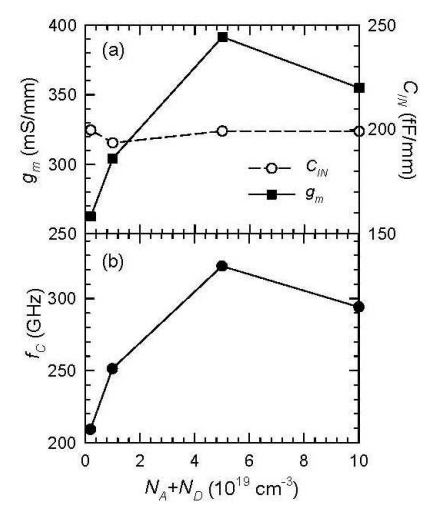

Fig. 3. Intrinsic MC values of (a) $g_{\mathrm{m}}$ and $C_{\mathrm{IN}}$, and (b) $f_{\mathrm{C}}$ as a function of $N_{\mathrm{A}}+N_{\mathrm{D}}$, for $V_{\mathrm{GDIFF}}=0 \mathrm{~V}$, $V_{\mathrm{DS}}=0.5 \mathrm{~V}$ and $V_{\mathrm{GOFF}}$ providing the maximum of $f_{\mathrm{C}}$. $L_{\mathrm{g}}=100 \mathrm{~nm}$ and $d_{\mathrm{C}}=40 \mathrm{~nm}$.

In order to diminish $C_{\mathrm{IN}}$, the distance between the gate electrodes must be increased, for example, by enlarging the channel width $d_{\mathrm{C}}$. Figure 4 presents the MC intrinsic 
results for different values of $d_{\mathrm{C}}$, showing that a wider channel provides also an improvement in the value of $g_{\mathrm{m}}$, i.e., a better control on the transfer of electrons between channels. Nevertheless, the improvement of $g_{\mathrm{m}}$ and $f_{\mathrm{C}}$ saturates for a value of about $d_{\mathrm{C}}=80 \mathrm{~nm}$.

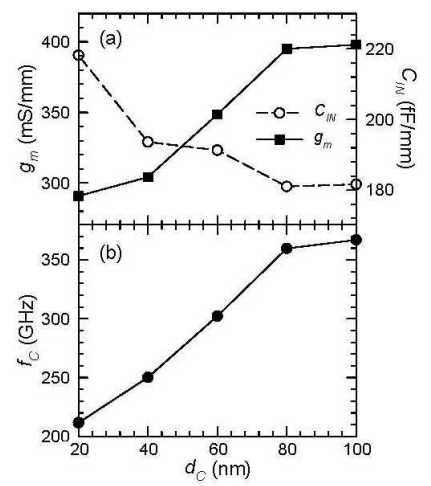

Fig. 4. Intrinsic MC values of (a) $g_{\mathrm{m}}$ and $C_{\mathrm{IN}}$, and (b) $f_{\mathrm{C}}$ as a function of $d_{\mathrm{C}}$, for $V_{\mathrm{GDIFF}}=0 \mathrm{~V}, V_{\mathrm{DS}}=0.5 \mathrm{~V}$, and $V_{\mathrm{GOFF}}$ providing the maximum of $f_{\mathrm{C}} . L_{\mathrm{g}}=100 \mathrm{~nm}$ and $N_{\mathrm{A}}+N_{\mathrm{D}}=10^{19} \mathrm{~cm}^{-3}$.

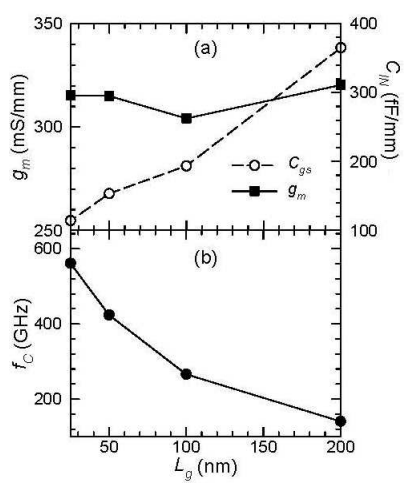

Fig. 5. Intrinsic MC values of (a) $g_{\mathrm{m}}$ and $C_{\mathrm{IN}}$, and (b) $f_{\mathrm{C}}$ as a function of $L_{\mathrm{g}}$, for $V_{\mathrm{GDIFF}}=0 \mathrm{~V}, V_{\mathrm{DS}}=0.5 \mathrm{~V}$, and $V_{\mathrm{GOFF}}$ providing the maximum of $f_{\mathrm{C}} \cdot d_{\mathrm{C}}=40 \mathrm{~nm}$ and $N_{\mathrm{A}}+N_{\mathrm{D}}=10^{19} \mathrm{~cm}^{-3}$.

Finally, in Fig. 5 we show that the most appropriate way to reduce $C_{\mathrm{IN}}$ is shortening the gate length $L_{\mathrm{g}}$. A shorter $L_{\mathrm{g}}$ does not lead to a reduction of $g_{\mathrm{m}}$ (neither to an increase as for traditional FETs), while the value of $C_{\text {IN }}$ decreases and thus $f_{\mathrm{C}}$ is remarkably enhanced. As a consequence, because of the different scaling rules of the VMT and the immunity to short-channel effects of the DG architecture [5], the limit for attaining high frequencies of operation comes from the technological difficulties met to achieve a perfect alignment of both gates of so small length.

\section{Conclusions}

In this work we have analyzed, by means of a $2 \mathrm{D}$ ensemble MC simulator, an InAlAs/InGaAs short-channel
VMT based on the DG-HEMT topology. By changing the gate voltages in differential mode, electrons are shifted between two channels with significantly different mobilities. The low values taken by $g_{\mathrm{m}}$, as well as the high capacitance between the gates $C_{\mathrm{g} 1 \mathrm{~g} 2}$ in DM operation, leads to a deficient dynamic behavior. However, the cut-off frequency of VMTs can be increased by rising the compensated doping $N_{\mathrm{A}}+N_{\mathrm{D}}$ and by enlarging the distance between the gate electrodes. However, given the immunity to short-channel effects of the DG architecture, the most appropriate way to reduce $C_{\mathrm{IN}}$ without lowering $g_{\mathrm{m}}$ (thus enhancing $f_{\mathrm{C}}$ ) is shortening the gate length $L_{\mathrm{g}}$.

\section{Acknowledgments}

This work has been partially supported by the European Commission through the ROOTHz Project ICT-2009-243845, by the Dirección General de Investigación (MEC) and FEDER through project TEC2007-61259/ MIC and by the Consejería de Educación, Junta de Castilla y León through projects SA019A08 and GR270.

\section{References}

[1] S. Tiwari, Compound Semiconductor Device Physics, Academic, New York 1992.

[2] N. Wichmann, I. Duszynski, X. Wallart, S. Bollaert, A. Cappy, IEEE Electron. Dev. Lett. 25, 354 (2004).

[3] N. Wichmann, I. Duszynski, S. Bollaert, J. Mateos, X. Wallart, A. Cappy, in: IEDM Tech. Dig. IEEE Cat. No. 04CH37602, 2004, p. 1023.

[4] N. Wichmann, I. Duszynski, X. Wallart, S. Bollaert, A. Cappy, IEEE Electron. Dev. Lett. 26, 601 (2005).

[5] B.G. Vasallo, N. Wichmann, S. Bollaert, Y. Roelens, A. Cappy, T. González, D. Pardo, J. Mateos, IEEE Trans. Electron Dev. 54, 2815 (2007).

[6] B.G. Vasallo, N. Wichmann, S. Bollaert, Y. Roelens, A. Cappy, T. González, D. Pardo, J. Mateos, IEEE Trans. Electron Dev. 55, 1535 (2008).

[7] N. Wichmann, B.G. Vasallo, S. Bollaert, Y. Roelens, X. Wallart, A. Cappy, T. González, D. Pardo, J. Mateos, Appl. Phys. Lett. 94, 103504 (2009).

[8] B.G. Vasallo, N. Wichmann, S. Bollaert, Y. Roelens, A. Cappy, T. González, D. Pardo, J. Mateos, IEEE Trans. Electron Dev., to be published.

[9] H. Sakaki, Jpn. J. Appl. Phys. 21, L381 (1982).

[10] K. Hirakawa, H. Sakaki, J. Yoshino, Phys. Rev. Lett. 54, 1279 (1985).

[11] K. Maezawa, T. Mizutani, M. Tomizawa, Jpn. J. Appl. Phys. 31, 757 (1992).

[12] K. Maezawa, T. Mizutani, IEEE Trans. Electron Dev. 39, 2438 (1992).

[13] K.J. Webb, E, B, Cohen, M.R. Melloch, IEEE Trans. Electron Dev. 48, 2701 (2001).

[14] M. Prunnila, J. Ahopelto, K. Henttinen, F. Gamiz, Appl. Phys. Lett. 85, 5442 (2004).

[15] C. Sampedro, F. Gamiz, A. Godoy, M. Prunnila, J. Ahopelto, Appl. Phys. Lett. 86, 202115 (2004).

[16] J. Mateos, T. González, D. Pardo, V. Hoël, A. Cappy, IEEE Trans. Electron Dev. 47, 1950 (2000). 